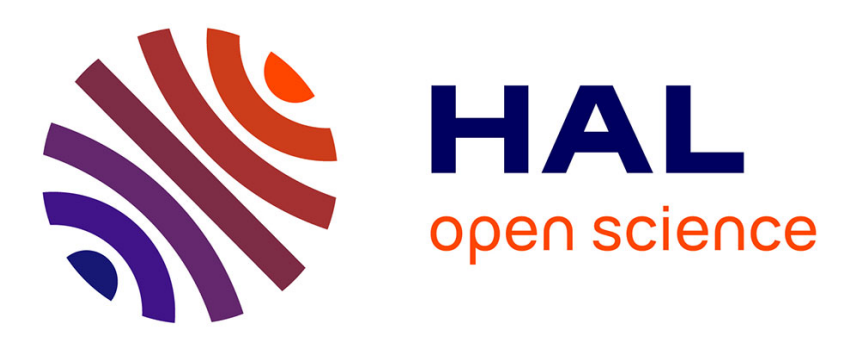

\title{
Discrete modelling of the fragmentation of granular media under compressive forces: Application to grinding process
}

Adolphe Kimbonguila, Mohamed Guessasma, Patrice Coorevits, Jérôme Fortin, Emmanuel Bellenger

\section{To cite this version:}

Adolphe Kimbonguila, Mohamed Guessasma, Patrice Coorevits, Jérôme Fortin, Emmanuel Bellenger. Discrete modelling of the fragmentation of granular media under compressive forces: Application to grinding process. The 6th International Conference on Engineering Computational Technology, ECT 2008, Sep 2008, Athens, Greece. hal-01095800

\section{HAL Id: hal-01095800 \\ https://hal.science/hal-01095800}

Submitted on 16 Dec 2014

HAL is a multi-disciplinary open access archive for the deposit and dissemination of scientific research documents, whether they are published or not. The documents may come from teaching and research institutions in France or abroad, or from public or private research centers.
L'archive ouverte pluridisciplinaire HAL, est destinée au dépôt et à la diffusion de documents scientifiques de niveau recherche, publiés ou non, émanant des établissements d'enseignement et de recherche français ou étrangers, des laboratoires publics ou privés. 


\title{
Discrete modelling of the fragmentation of granular media under compressive forces: Application to grinding process
}

\author{
A. Kimbonguila ${ }^{1}$, M. Guessasma ${ }^{1}$, P. Coorevits ${ }^{1}$, J. Fortin $^{2}$, E. Bellenger ${ }^{1}$ \\ UPJV- LTI EA 3899 \\ ${ }^{1}$ IUT de l'Aisne, 48 rue d'Ostende, F-02100 Saint-Quentin, France \\ ${ }^{2}$ INSSET, 48 rue Raspail, F-02100 Saint-Quentin, France
}

\begin{abstract}
The aim of this work is a numerical simulation of physical phenomena during the grinding process by using a discrete approach. The Discrete Element Method (DEM) has been used to model the fragmentation and mechanical behaviour of granular packing under compressive forces. Through this study, we show the effect of contact force chains on the stress field distribution into the granular packing. We have also studied the fragmentation mode of grains induced by the stress field and its influence on the granulometry. Numerical simulations of granular packing subjected to compressive loading have been carried out with MULTICOR software.
\end{abstract}

Keywords: Granular media, DEM, fragmentation, grinding process.

\section{Introduction}

The grinding process is commonly used in many industrial fields such as: pharmaceutical, mineral or food. However, such a process doesn't come cheap, essentially due to the energy cost and to the low quality of finished products (heterogeneity of grain size and shape). Hence, the economic stakes are large and the optimization of such process seems crucial. Due to this energy cost, understanding the grinding process for granular media under compressive forces could be a good way to reach a better fragmentation efficiency. In order to be able to get answers to many questions about the grinding process, numerical simulations seem to be a good way to predict the mechanical behaviour of granular media. To carry out such simulations, we must define the fragmentation mode of one grain subjected to a random contact forces distribution induced by the adjacent grains. The investigations carried out on the fracture of grain [1-6] have shown that the fragmentation mechanism depends on several parameters, which are: the size, the shape, the material properties and the loading configuration. It has been concluded that the crack into the grain follows a transversal propagation leading to the 
fragmentation. In addition, the crack propagation depends on the stress field inside the grain [1] and the stress intensity factor of material [1-4].

Throughout this study, we have developed a discrete modelling for granular packing subjected to compressive loading by using DEM. We have used a grain fragmentation model depending on contact forces randomly spread [4,6]. The criterion of fracture depends on the tensile stress inside the grain and on the stress intensity factor of the material. The proposed model of fragmentation has been implemented in MULTICOR software [7] and carried out for numerical calculations of granular packing under œdometric compression. Through this mechanical test, the objective is to be able to reproduce similar effects arising during grinding process.

\section{DEM approach}

The 2D modelling of granular material is considered as a stacking of several discs subjected to a given mechanical solicitation. However, the discs are commonly called particles or grains in the literature. The conventional DEM allows to model really deformable particles as well as complex shapes (from the ellipsoid to the polygon) [8-10]. Here, we have studied the simple case of non-deformable and non penetrable particles in 2D with the computational software MULTICOR. The coordinates and the rotations of Euler are the configuration parameters $q$. The gyroscopic and centrifugal terms are equal to zero in $2 \mathrm{D}$. The matrix of generalized mass $M$ of the system doesn't depend on $q$ that is diagonal block. The mechanical equation (1) can be written in the following form:

$$
M \ddot{q}=F_{\text {ext }}(q, \dot{q}, t)+R^{\alpha}
$$

where $F_{\text {ext }}$ represents the known external forces and $R^{\alpha}$ the unknown interior forces related to contact reactions and $\alpha$ the contact number.

In a system composed of $p$ heterogeneous particles, the critical parameter for the modelling time is the maximum number of interactions between particles. The more the interaction range is important the more we have to test the possible interactions between particles (Figure 1). MULTICOR uses the partitioning method coupled to a connectivity table. This technique allows to reduce considerably the computational time. In that case, the computational time no longer increases like $O\left(p^{2}\right)$ but only like $O(p)$, which is almost optimal [11]. For each couple of particles $\Omega_{i}$ and $\Omega_{j}$ which may enter in contact, is associated with a local reference whose axes are oriented according to the two unit vectors $n$ and $t$, respectively normal and tangential vectors in the contact plan. The normal $n$ is directed from $\Omega_{i}$ and $\Omega_{j}$. The variables put in duality are $\dot{u}^{i j}$, the relative local velocity of $\Omega_{i}$ compared to $\Omega_{j}$, and the contact reaction $r^{i j}$ of $\Omega_{i}$ and $\Omega_{j}$. In the local base, they are written by equations (2):

$$
\dot{u}^{i j}=\dot{u}_{t}^{i j}+\dot{u}_{n}^{i j} n, \quad r^{i j}=r_{t}^{i j}+r_{n}^{i j} n
$$


where $\dot{u}_{n}^{i j}$ is the normal separation velocity, $\dot{u}_{t}^{i j}$ the sliding velocity, $r_{n}^{i j}$ the contact pressure and $r_{t}^{i j}$ the adherence force.

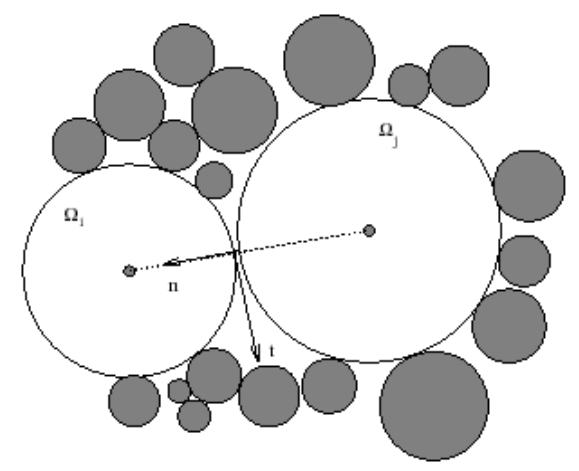

Figure 1: Detection of contact.

The introduction of Coulomb's friction $\mu$ leads to a non linear problem which can't be solved by a linear programming method. Unlike the usual approach, the bipotential method leads to a single variational principle and an inequality [12]. By using Usawa's algorithm, we obtain a resolution algorithm of the constitutive law based on the predictive-corrective scheme expressed by equations (3):

$$
\begin{aligned}
& \text { predictor : } \tau^{i j}=r^{i j}-\gamma\left[\dot{u}_{t}^{i j}+\left(\dot{u}_{n}^{i j}+\mu\left\|-\dot{u}_{t}^{i j}\right\|\right) \cdot n\right], \\
& \text { corrector : } r^{i j}=\operatorname{proj}\left(\tau^{i j}, K_{\mu}\right)
\end{aligned}
$$

where $\gamma$ is a numerical parameter and $K_{\mu}$ the Coulomb's cone.

The projection of the prediction $\tau^{i j}$ on the Coulomb's cone $K_{\mu}$ leads to one of these states: non contact, contact with adherence or sliding contact. Conventionally, at each time step, the contact forces in the system are determined repeatedly by the method of successive balances based on a Gauss-Seidel algorithm for the $2 D$ version. Each contact force is calculated by adopting temporary values over the other contacts. The convergence is obtained when the force confirms the unilateral contact law with dry friction.

\section{Stress tensor of granular material}

In order to understand the mechanisms governing the fragmentation of the grains subjected to compressive loading, we must be able to compute the stress tensor inside each grain of the discrete media. The knowledge of stress field leads to the prediction of the grain fragmentation according to the material strength and the fracture criterion.

Previous studies [13-14] have been carried out in order to determine the stress tensor of granular material. The proposed stress tensor is averaged with respect to a 
Representative Elementary Volume (REV) containing $N_{p}$ grains separated by voids (Figure 2).

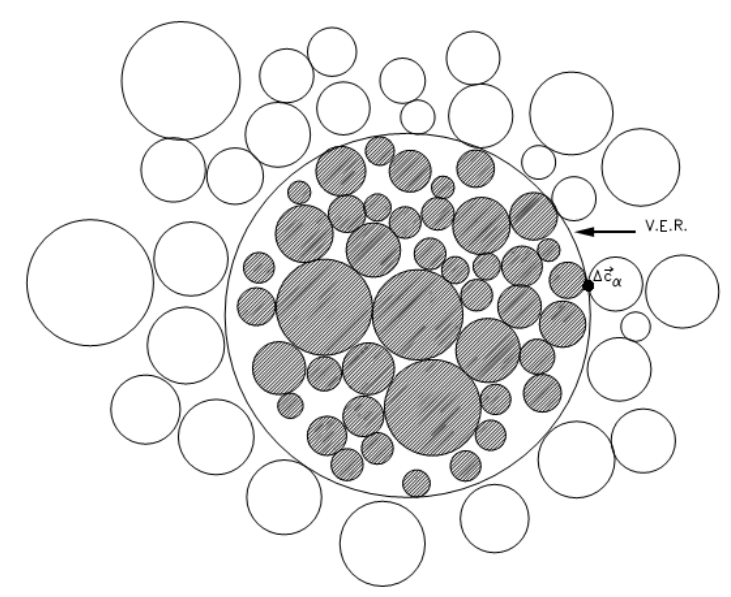

Figure 2: REV of granular material.

The grains are considered as rigid solids in punctual contact for each grains $p$ occupying the volume $V_{p}$, the expression of stress tensor is given by the following equation (4):

$$
\sigma_{p}=\frac{1}{V_{p}}\left[\int_{V_{p}} q \otimes \rho(g-\ddot{q}) d V+\sum_{\alpha} q_{c \alpha} \otimes r_{\alpha}\right]
$$

where $g$ denote the gravity, $q$ the position vector, $\ddot{q}$ its acceleration and $r_{\alpha}$ the contact reaction acting on the particle at the point of contact $q_{c \alpha}$. According to the additivity on internal moments in the sense of Moreau [15], the mean stress tensor $\sigma$ on the $R E V$ can be written as follows (5):

$$
\sigma=\frac{1}{V} \sum_{p \in V} V_{p} \sigma_{p}
$$

Therefore, the stress tensor $\sigma_{p}$ is composed in three parts: contact reaction stress, gravity stress and stress part due to the inertial forces. Accordingly, the contribution of inertial forces insures the symmetry of the stress tensor in the sense of Cauchy.

In the present study, the stress tensor will be computed with respect to a REV volume containing only one grain. The stress tensor will be then determined inside each grain by considering only the contribution of contact forces (inertial and gravity effects are neglected). 


\section{Numerical modelling of grain fragmentation}

The numerical modelling carried out in this study describes the mechanical behaviour of granular packing under compressive loading and the fragmentation mechanisms which brought in play by the contact force chains.

If we consider a single grain submitted to uniaxial compression by considering Hertz's assumptions [16], the analytical resolution permits to verify the existence of tensile stresses localised at the grain centre and perpendicular to the loading direction [17]. Due to the existence of these traction forces, the first cracks start at the centre of the grain leading to the propagation of a transversal fracture following the direction of compression force. In the case of one grain subjected to many contact forces randomly spread, the loading on the grain can be considered as a biaxial compression following the principal direction of stress field as shown in Figure 3 . The tensile stress inside the grain is then computed by taking into account the contribution of compression forces $r_{\max }$ and $r_{\min }$.

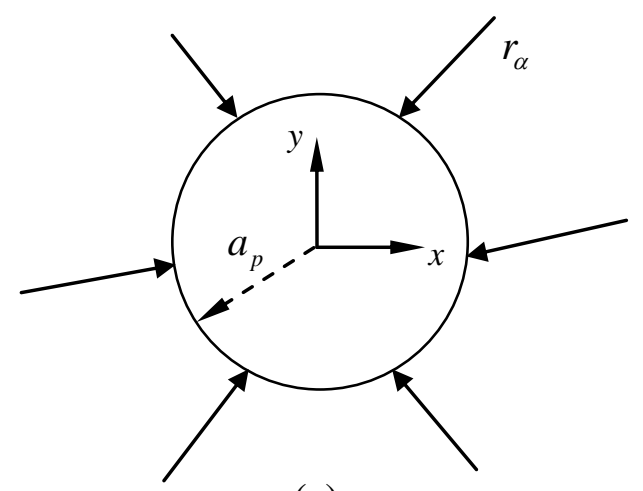

(a)

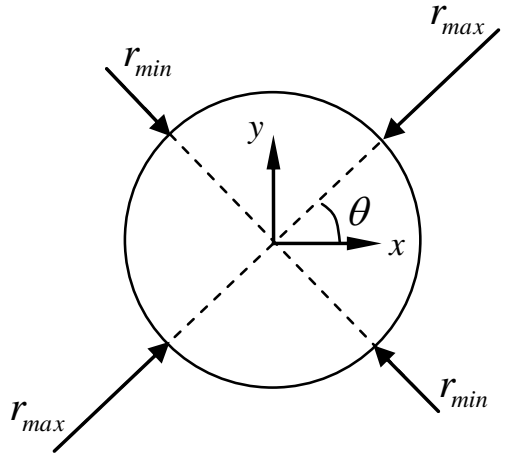

(b)

Figure 3: (a) Contact forces randomly spread - (b) Biaxial compression.

\subsection{Mechanical formulation}

In 2D dimensions, the stress tensor inside the grain (6), resulting from the contact forces (Figure 3(a)), is given by the following relationship:

$$
\sigma_{p}=\frac{a_{p}}{V_{p}} \sum_{\alpha} n_{c \alpha} \otimes r_{\alpha}
$$

with $r_{\alpha}$ the contact forces on the grain, $\alpha$ the number of contact forces, $a_{p}$ the grain radius, $V_{p}$ the unitary volume of grain and $n_{c \alpha}$ the unit normal vector.

The stress tensor inside the grain can be decomposed in two parts (7). The first one is represented by the hydrostatique stress tensor, while the second one is expressed by the deviatoric stress tensor: 


$$
\sigma_{p}=\frac{\sigma_{\max }+\sigma_{\min }}{2}\left(\begin{array}{ll}
1 & 0 \\
0 & 1
\end{array}\right)+\frac{\sigma_{\max }-\sigma_{\min }}{2}\left(\begin{array}{cc}
\cos 2 \theta & \sin 2 \theta \\
\sin 2 \theta & -\cos 2 \theta
\end{array}\right)
$$

where $\sigma_{\max }$ and $\sigma_{\min }$ are respectively the major and minor principal stresses and $\theta$ the principal direction with respect to the horizontal axe.

The principal stresses $\sigma_{\max }, \sigma_{\min }$ and the principal direction $\theta$ are given respectively by the following relations (8):

$$
\begin{gathered}
\sigma_{\text {max } \text { min }}=\frac{\sigma_{x x}+\sigma_{y y}}{2} \pm \sqrt{\left(\frac{\sigma_{x x}-\sigma_{y y}}{2}\right)^{2}+\sigma_{x y} \sigma_{y x}} \\
\theta=\frac{1}{2} \arctan \left(\frac{2 \sigma_{x y}}{\sigma_{x x}-\sigma_{y y}}\right)
\end{gathered}
$$

where $\sigma_{x x}$ and $\sigma_{y y}$ are respectively the tensile stresses according to the $x$ and $y$ axis and $\sigma_{x y}$ is the in-plane shear stress.

By considering the case of one grain subjected to biaxial compression, the principal stresses $\sigma_{\max }$ and $\sigma_{\min }$ are written as follows (9):

$$
\sigma_{\max , \min }=\frac{2 r_{\max , \min }}{\pi a_{p}}
$$

where $r_{\max }$ and $r_{\min }$ are respectively the compression forces following the major and minor directions.

Regarding the state of stress at the centre of grain, the analytic solution [16] leads to the following expressions (10):

$$
\begin{aligned}
\sigma_{\text {tensile }}^{c} & =\frac{r}{\pi a_{p}} \\
\sigma_{\text {compression }}^{c} & =\frac{-3 r}{\pi a_{p}}
\end{aligned}
$$

where $\sigma_{\text {tensile }}^{c}$ and $\sigma_{\text {compression }}^{c}$ are respectively the tensile and compression stresses at the grain centre. 
For the case of biaxial compression (Figure 3), the state of stress at the grain centre is obtained by considering the contribution of the both compression forces $\left(r_{\max }\right.$ and $\left.r_{\min }\right)$. The following relationships (11) express the tensile and compression stresses, respectively $\sigma_{\text {tensile }}^{c}$ and $\sigma_{\text {compression }}^{c}$ :

$$
\begin{aligned}
& \sigma_{\text {tensile }}^{c}=\frac{r_{\text {max }}-3 r_{\text {min }}}{\pi a_{p}}=\frac{\sigma_{\text {max }}-3 \sigma_{\text {min }}}{2} \\
& \sigma_{\text {compression }}^{c}=\frac{r_{\text {min }}-3 r_{\max }}{\pi a_{p}}=\frac{\sigma_{\text {min }}-3 \sigma_{\max }}{2}
\end{aligned}
$$

The tensile stress $\sigma_{\text {tensile }}^{c}$ is responsible of the beginning of cracks at the grain centre and its propagation following the major principal direction (direction of $r_{\max }$ force). Therefore, when $\sigma_{\text {tensile }}^{c}$ overcomes a given critical stress, the fracture of grain can be started. Many experimental studies have leaded to several expressions of critical stress depending on the stress intensity factor, the geometric and problem dimensions $[4,18]$.

The force leading to the fracture of one grain, $r_{\text {fracture }}$, is given by the following equation (12):

$$
r_{\text {fracture }}=K_{I}\left(2 a_{p}\right)^{\alpha} ; \alpha=d-3 / 2
$$

where $K_{I}$ is the mode $I$ stress intensity factor ( $I$ : tensile mode), $a_{p}$ is the grain radius and $d$ is the problem dimension $(d=2$ in $2 D)$.

By using the relation (10) expressing the tensile stress, the critical stress from which the fracture of grain is reached can be written in the following form (13):

$$
\sigma_{\text {fracture }}=\frac{r_{\text {fracture }}}{\pi a_{p}}=\sqrt{\frac{2}{a_{p}}} \times \frac{K_{I}}{\pi}
$$

Hence, when the tensile stress $\sigma_{\text {tensile }}^{c}$ at the centre of grain overcomes the critical stress $\sigma_{\text {fracture }}$, we have fracture of grain induced by traction.

However, we haven't taken into account in the proposed approach other phenomena which can occur during the grinding process. Indeed, some effects like attrition or heat dissipation (by friction and shock) have certainly an influence on the granular material behaviour during the grinding process. 


\subsection{Numerical model of grain fragmentation}

Some numerical models for the grain fragmentation have been carried out in many studies $[1,4,6]$. However, the principal deficiency of these models is the nonconservation of the grain mass at each fragmentation. Some solutions can be performed in order to reduce the percentage of loss mass. We can insert into the granular packing grains with small radius after each fragmentation. But, this solution isn't easy to perform due to the difficulty to satisfy the contact conditions between grains. Another way which can be used to make sure of the mass conservation is to consider grain density according to the time.

In our study, we have considered the fragmentation model as shown in Figure $4 \mathrm{~b}$ [4], with no mass conservation. The proposed model is performed with a loss of mass from approximately 13\%. Our works in progress will lead to reduce this percentage. The numerical model from which the fragmentation is taken into account is composed of 12 grains resulting from the subdivision of the initial grain. The grain fragmentation, following the principal directions (Figure 4(a)), will be performed as follows: the initial grain is divided in 4 gains with radius equal to $a_{p} / 2$ and $a_{p} / 3$, and 8 gains with radius equal to $a_{p} / 6$ and $a_{p} / 11$ (Figure $4 \mathrm{~b}$ ).
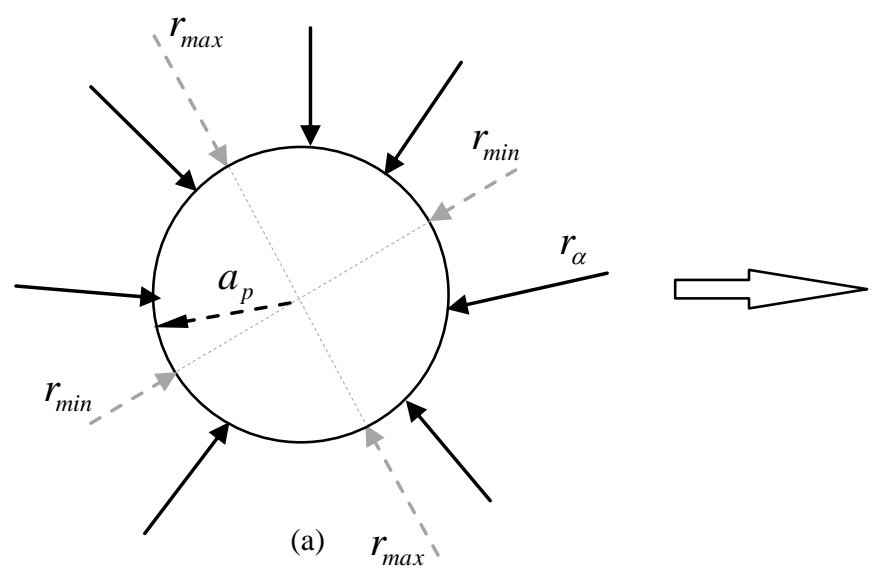

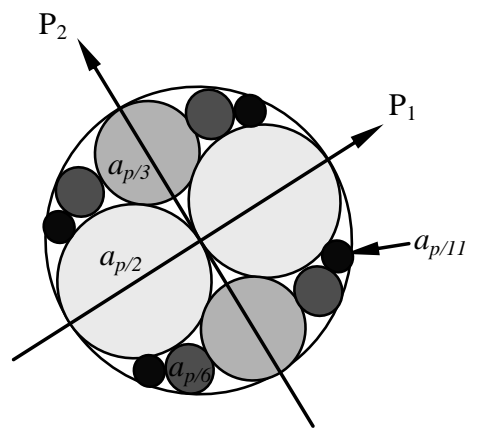

(b)

Figure 4: (a) Biaxial compression following the principal directions - (b) Numerical modelling of fragmentation.

The fragmentation grain is stopped for a critical size of grain. The relationship (14) gives the equation from which the critical grain radius $a_{c r i t}$ is computed:

$$
a_{\text {crit }}=\frac{K_{I}{ }^{2}}{\pi \sigma_{\text {elastic }}^{2}}
$$

where $K_{I}$ is the mode $I$ stress intensity factor and $\sigma_{\text {elastic }}$ the elastic strength of material. 


\section{Numerical simulations}

The numerical simulations have been carried out with MULTICOR software in order to perform calculations with the proposed model. We have considered a granular packing under compressive loading (Figure 5).

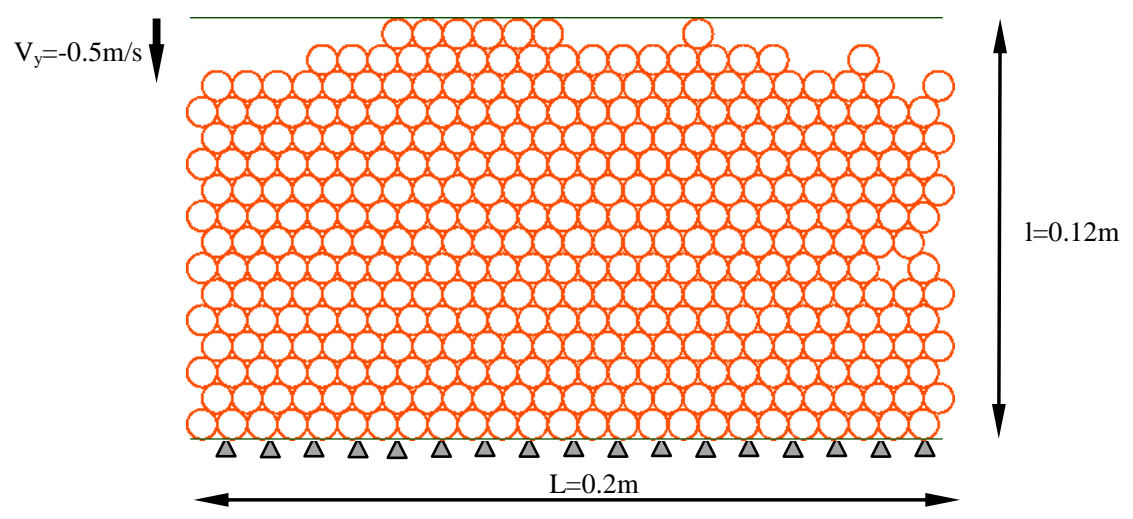

Figure 5: Oedometric test of granular packing.

The granular bed contains 371 grains with radius equal to $4 \mathrm{~mm}$. The granular material is perfectly rigid with: density equal to $1400 \mathrm{~kg} / \mathrm{m}^{3}$, friction coefficient equal to 0.2 and tensile strength about $150 \mathrm{MPa}$. The boundary conditions are the following: top wall velocity is imposed; bottom wall is maintained fixe; periodic conditions are imposed on the lateral sides (Figure 5).

Time $=2.510^{-3} \mathrm{~s}$
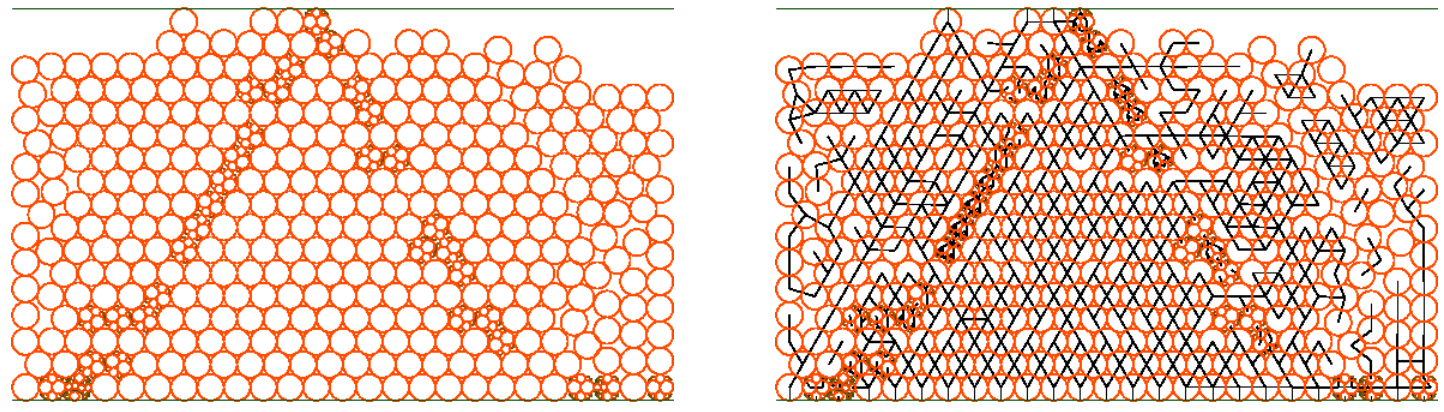

Time $=1.710^{-2} \mathrm{~s}$

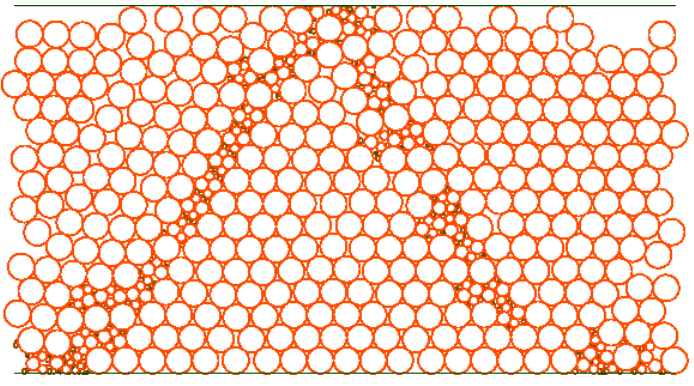

(a)

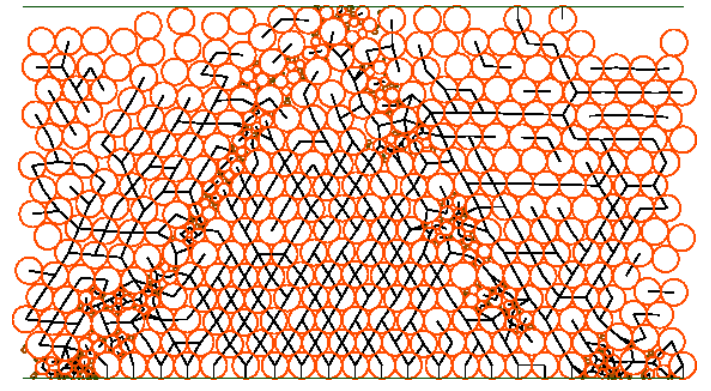

(b)

Figure 6: (a) Granular packing configurations - (b) Contact force chains. 
At each time step, we compute for each grain the traction stress at the centre. When the condition (13) is satisfied, the grain is substituted by the numerical model (Figure 4(b)). Figure 6 shows different configurations of the granular packing during the fragmentation process and the corresponding force chain distributions.

After an elapsed time of $2.510^{-3} \mathrm{~s}$, two shear bands appear into the granular packing inducing the fragmentation of grains across these bands. This phenomenon is often observed in the granular material subject to a large plastic deformation. At the next step (time $=1.710^{-2} \mathrm{~s}$ ), the granular packing becomes less rigid leading to a lowest stress field inside the grains. During the oedometric test, segregation phenomenon has been observed with respect to the grain size. The new configuration (time $=2.510^{-2} \mathrm{~s}$ ) of the granular bed shows that the small grains are subjected to a quasi-uniaxial compression because of their few contact forces number (Figure 7).

Time $=2.510^{-2} \mathrm{~s}$
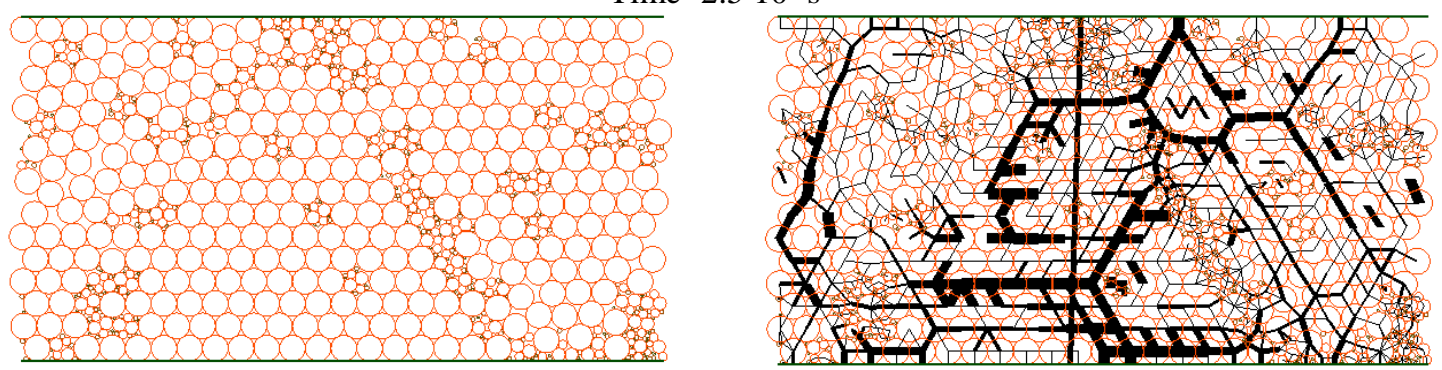

Figure 7: Influence of the granulometry during the grinding process.

The graph given below provides information about the traction stress evolution into the granular packing during the grinding process. The stress values have been plotted with respect to the grain position (Figure 8). The maximum value reached by the traction stress at different position means that we have fragmentation of grain.

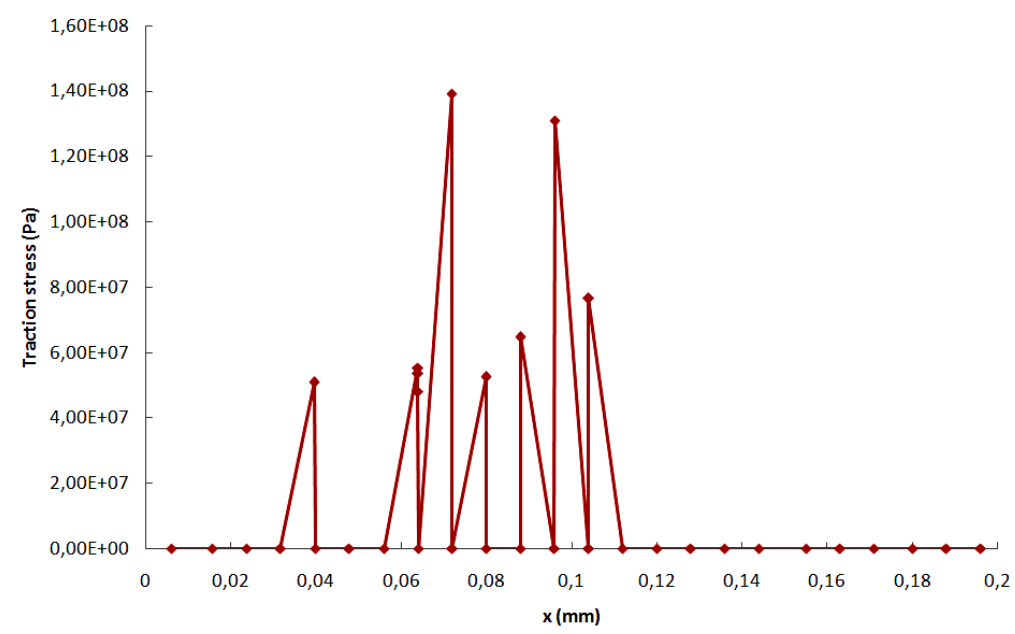

Figure 8: Traction stress with respect to the grain position. 
From these observations, the fragmentation is easier for the small grains than for the large grains during the grinding process. Indeed, the large grains are subjected to several contact forces inducing a quasi-hydrostatic effect, which reduces the possibility to start the fragmentation mechanism. In conclusion, the fragmentation process of grain depends of both parameters: the grain size and the stress state inside the grain.

\section{Conclusion}

Throughout the present paper, we have investigated the mechanisms which brought in play by contact force chains during the grinding process by compression of granular material. In fact, the loading induces by this process type leads to traction forces inside the grain, which are responsible of the grain fracture. A numerical model of fragmentation has been performed by using criterion based on the stress intensity factor. The numerical simulations of oedometric test carried out with the proposed model have shown the influence of the grain size and the stress state on the fragmentation mechanism. Our works in progress will lead to better models in order to reduce the percentage of mass loss and taking into account other mechanical effects.

\section{References}

[1] J.A. Aström, H.J. Herrmann, "Fragmentation of grains in a two-dimensional packing”, Eur. Phys. J., B(5), 551-554, 1998.

[2] H. Morikawa, "Analytical studies on local damage to reinforced concrete structures under impact loading by discrete element method", Nuclear Eng. and Design, 179, 157-177, 1998.

[3] O. Tsoungui, D. Vallet, J.C. Charmet, "Use of contact area trace to study the force distributions inside 2D granular systems", Granular Matter 1, 65-69, 1998.

[4] O. Tsoungui, D. Vallet, J.C. Charmet, "Numerical model of crushing of grains inside two-dimensional branular materials", Powder Technology, 105, 190198, 1999.

[5] J.P. Morris, M.B. Rubin, G.I. Block, M.P. Bonner, "Simulations of fracture and fragmentation of geologic materials using combined FEM/DEM analysis", Int. J. of Impact Engineering, 33, 463-473, 2006.

[6] S. Lobo-Guerrero, L.E. Vallejo, "Influence of pile shape and pile interaction on the crushable behaviour of granular material around driven piles: DEM analyses", Granular Matter, 9, 241-250, 2007.

[7] J. Fortin, O. Millet, G. de Saxce, "Numerical simulation of granular materials by an improved discrete element method", Int. J. for Num. Meth. in Eng., 62, 639-663, 2004. 
[8] Cundall, P. A., and O.D.L. Strack. "The Development of Constitutive Laws for Soil Using the Distinct Element Method", Numerical Methods in Geomechanics, 1, 289-317, 1979.

[9] P.A. Cundall, O.D.L. Strack. "Modeling of Microscopic Mechanisms in Granular Material", Mechanics of Granular Materials, 137-149, 1983.

[10] B. Cambou, A; Mahboudi, J.J. Fry, "Numerical modelling of the mechanical behavior of non spherical crushable particles", Powders and Grains, Behringer and Jenkins, 1997.

[11] J. Fortin, P. Coorevits, "Selecting contact particles in dynamics granular mechanics systems", J. of Comp. and App. Math., 168, 207-213, 2004.

[12] J. Fortin, G. De Saxce, "Modelisation numerique des milieux granulaires par l'approchedu bi-potentiel", C. R. Acad. Sci., 327, 721-724, 1999.

[13] J. Fortin, O. Millet, G. de Saxce, "Construction of an average stress tensor for a granular medium”, Eur. J. of Mech., A/Solids, 22, 567-582, 2003.

[14] M. Guessasma, J. Fortin, E. Bellenger, "Numerical modelling of mechanical tests using discrete element method”, CST’06, Las Palmas, Spain, 12-15 Sep., 2006.

[15] J.J. Moreau, "Numerical investigation of shear zones in granular materials, In: Grassberger", Proc. HLRZ-Workshop on Friction, Arching, Contact Dynamics. World Scientific, Singapore, 233-247, 1997.

[16] K.L. Johnson,"Contact Mechanics", Cambridge Univ. Press, Cambridge, 1985.

[17] R. Kienzler, W. Schmitt, "Powder Technology", 611990.

[18] A.W. Larson, "The effect of size and contact geometry in single particle crushing", Master's thesis, MIT, Boston, 1986. 